physics/0609017

\title{
Scalar Top Study: Detector Optimization
}

\author{
C. Milsténe ${ }^{1}$, A. Sopczak ${ }^{2}$ \\ ${ }^{1}$ Fermilab, USA; ${ }^{2}$ Lancaster University, UK
}

\begin{abstract}
A vertex detector concept of the Linear Collider Flavour Identification (LCFI) collaboration, which studies pixel detectors for heavy quark flavour identification, has been implemented in simulations for c-quark tagging in scalar top studies. The production and decay of scalar top quarks (stops) is particularly interesting for the development of the vertex detector as only two c-quarks and missing energy (from undetected neutralinos) are produced for light stops. Previous studies investigated the vertex detector design in scenarios with large mass differences between stop and neutralino, corresponding to large visible energy in the detector. In this study we investigate the tagging performance dependence on the vertex detector design in a scenario with small visible energy for the International Linear Collider (ILC).
\end{abstract}

Presented at the 2006 International Linear Collider Workshop - Bangalore, India,

to be published in the proceedings. 



PRAMANA
$\begin{array}{r}\text { journal of } \\ \text { physics }\end{array}$

pp. $1-5$

\title{
Scalar Top Study: Detector Optimization
}

\author{
C. Milsténe ${ }^{1}$ and A. Sopczak ${ }^{2}$ \\ ${ }^{1}$ Fermilab, USA; ${ }^{2}$ Lancaster University, UK
}

\begin{abstract}
A vertex detector concept of the Linear Collider Flavour Identification (LCFI) collaboration, which studies pixel detectors for heavy quark flavour identification, has been implemented in simulations for c-quark tagging in scalar top studies. The production and decay of scalar top quarks (stops) is particularly interesting for the development of the vertex detector as only two c-quarks and missing energy (from undetected neutralinos) are produced for light stops. Previous studies investigated the vertex detector design in scenarios with large mass differences between stop and neutralino, corresponding to large visible energy in the detector. In this study we investigate the tagging performance dependence on the vertex detector design in a scenario with small visible energy for the International Linear Collider (ILC).
\end{abstract}

\section{Introduction}

The development of a vertex detector for a Linear Collider is an important and challenging enterprise. A key aspect is the distance of the innermost layer to the interaction point, which is related to radiation hardness and beam background. Another key aspect is the number of radiation lengths the particles go through, since it determines the multiple scattering which affects the vertex reconstruction.

The optimization of the vertex detector tagging performance is of great importance for studies of physics processes. While mostly at previous and current accelerators (e.g. SLC, LEP, Tevatron) b-quark tagging has revolutionized many searches and measurements, cquark tagging will be very important at a future Linear Collider, for example, in studies of Supersymmetric dark matter [1]. Therefore, c-quark tagging could be a benchmark for vertex detector developments. The scalar top production and decay process, and the implemented vertex detector geometry are shown before [2].

The analysis for a large mass difference with the SPS-5 parameter point (ISAJET) $m_{\tilde{\mathrm{t}}_{1}}=$ $220.7 \mathrm{GeV}, m_{\tilde{\chi}_{1}^{0}}=120.0 \mathrm{GeV}$ and $\cos \theta_{\tilde{\mathrm{t}}}=0.5377$ was previously performed [3]. For $25 \%$ (12\%) efficiency 3800 (1800) signal events and 5400 (170) background events without c-quark tagging were obtained, while the background was reduced to 2300 (68) events with c-quark tagging.

The vertex detector radiation length was varied between single thickness (TESLA TDR) and double thickness. In addition, the number of vertex detector layers was varied between 5 layers (innermost layer at $1.5 \mathrm{~cm}$ as in the TESLA TDR) and 4 layers (innermost layer at $2.6 \mathrm{~cm}$ ). For SPS-5 parameters the following numbers of background events remain [3]:

\begin{tabular}{l|c|cc} 
Thickness & layers & $12 \%$ signal efficiency & $25 \%$ signal efficiency \\
\hline Single & $5(4)$ & $68(82)$ & $2300(2681)$ \\
Double & $5(4)$ & $69(92)$ & $2332(2765)$
\end{tabular}




\section{Milsténe and A. Sopczak}

A significant larger number of background events was expected if the first layer of the vertex detector is removed. The distance of the first layer to the interaction point is also an important aspect from the accelerator physics (beam delivery) perspective. The interplay between the beam delivery and vertex detector design in regard to critical tolerances like hardware damage of the first layer and occupancy (unable to use the data of the first layer) due to beam background goes beyond the scope of this study and will be addressed in the future.

For large visible energy (large mass difference) no significant increase in the expected background was observed for doubling the thickness of the vertex detector layers [3,4]. In this study the effect of the vertex detector design for events with smaller visible energy in the detector is addressed.

\section{Signal and Background Simulations}

The production of simulated light stops at a $500 \mathrm{GeV}$ Linear Collider is analyzed using high luminosity $\mathcal{L}=500 \mathrm{fb}^{-1}$. The signature for stop pair production at an $\mathrm{e}^{+} \mathrm{e}^{-}$collider is two charm jets and large missing energy:

$$
\mathrm{e}^{+} \mathrm{e}^{-} \rightarrow \tilde{\mathrm{t}}_{1} \overline{\tilde{\mathrm{t}}}_{1} \rightarrow \mathrm{c} \tilde{\chi}_{1}^{0} \overline{\mathrm{c}} \tilde{\chi}_{1}^{0} .
$$

For small $\Delta m=m_{\tilde{\mathrm{t}}_{1}}-m_{\tilde{\chi}_{1}^{0}}$, the jets are relatively soft and separation from backgrounds is very challenging. Backgrounds arising from various Standard Model processes can have cross-sections that are several orders of magnitude larger than the signal. Thus, it is necessary to study this process with a realistic detector simulation. Signal and background events are generated with PYTHIA 6.129 [5], including a scalar top signal generation [6] previously used in Ref. [7]. The detector simulation is based on the fast simulation SiMDET [8], describing a typical ILC detector. Good agreement in comparisons with SGV [9] detector simulations was obtained $[3,4]$.

Cross-sections for the signal process and the relevant backgrounds have been computed with code used in Ref. [10] and by GRACE 2.0 [11], with cross-checks to COMPHEP 4.4 [12]. A minimal transverse momentum cut, $p_{\mathrm{t}}>5 \mathrm{GeV}$, is applied for the two-photon background, to avoid the infrared divergence. Details of the event selection are given in Ref. [1].

The c-tagging with the LCFI detector is based on the vertex identification and a neural network application [13]. The vertex identification considered three cases for each jet independently:

a) only a primary vertex. In this case, the two tracks with the largest separation in the $r$ $\phi$ plane are considered and for these tracks the neural network variables include, the impact parameter and its significance (impact parameter divided by uncertainty) both in the $r-\phi$ plane and in the $z$-direction, their momenta, and the joined probability in $r-\phi$ plane and $z$ direction.

b) one secondary vertex. In addition to the previous variables, the decay length significance of the secondary vertex, the multiplicity and momenta of all associated tracks, and the $P_{t}$ corrected mass of the secondary vertex (corrected for neutral hadrons and neutrinos), the $P_{t}$ of the decay products perpendicular to the flight direction between primary and secondary vertex, and the joint probability in $r-\phi$ and $z$-direction. 


\section{Linear Collider Workshop}

c) more than one secondary vertex. Two secondary vertices are considered, where the tracks are assigned to the vertex closest to the primary vertex, and the neural network input variables are defined as in case b).

The neural network is tuned with 255,000 simulated signal and 240,000 We $\nu$ background events. The signal events are a combination of all simulated signal events for the scalar top mass range between 120 to $220 \mathrm{GeV}$ and for $\Delta m=5,10$ and $15 \mathrm{GeV}$.

After a preselection which substantially reduces the background while keeping about $70 \%$ of the signal, six sequential cuts are applied: number of jets, missing energy, acollinearity, thrust angle, transverse momentum, and the jet-jet invariant mass and ctagging [1]. The background consists of the following processes $\mathrm{W}^{+} \mathrm{W}^{-}, \mathrm{ZZ}, \mathrm{We} \nu$, eeZ, $\mathrm{q} \overline{\mathrm{q}}(\mathrm{q} \neq \mathrm{t}), \mathrm{t} \overline{\mathrm{t}}$, and two-photon. After all cuts, the total background of about 5680 events is dominated by about $5044 \mathrm{We} \nu$ events [1]. A scalar top signal of $120 \mathrm{GeV}$ has been simulated with a neutralino mass of $110 \mathrm{GeV}$. The selection efficiency is about $20 \%{ }^{1}$ and 11,500 signal events are expected for a standard LCFI vertex detector configuration as given in the TESLA TDR.

\section{Varying the Vertex Detector Design}

This study of the vertex detector design is based on 50,000 simulated $120 \mathrm{GeV}$ signal and 210,000 We $\nu$ background events for each detector design. After preselection 29,842 signal and 53,314 We $\nu$ events are selected, corresponding to 34,318 and 779,450 events per $500 \mathrm{fb}^{-1}$, respectively. This preselection signal efficiency of $59.7 \%$ does not depend on the vertex detector design. Four detector designs are compared:

$\mathrm{VX}_{12}$ : the TESLA TDR design with 5 layers and single $\left(0.064 \% X_{0}\right.$ radiation length per layer).

$\mathrm{VX}_{22}$ : 4 layers (the innermost layer removed). This scenario could for example occur if the vertex detector is exposed to a large dose of machine background from the accelerator. The optimization of the radius of the innermost layer is an important aspect in the design of a vertex detector for a Linear Collider.

$\mathrm{VX}_{32}$ : 5 layers and double material thickness $\left(0.128 \% X_{0}\right.$ radiation length per layer). As the rigidity of the sensitive elements and the support structure is another important aspect in the detector design, the material budget has to be taken into account.

$\mathrm{VX}_{42}$ : 4 layers (the innermost layer removed) and double thickness $\left(0.128 \% X_{0}\right.$ radiation length per layer).

The c-tagging efficiency per event is normalized to the number of signal events after the preselection and requiring two jets. At least one c-tagged jet is required and the efficiency is given in Fig. 1 as a function of the purity, where purity is defined as the ratio of the

\footnotetext{
${ }^{1}$ The retuning of the c-tagging neural network increased the selection efficiency from $19 \%$ [1] to $20 \%$ and the We $\nu$ background from 5044 [1] to $5322 \pm 280$ events.
} 


\section{Milsténe and A. Sopczak}

number of simulated signal events after the c-tagging to all c-tagged events assuming the same luminosity for signal and background. The different purities are obtained by varying the cut on the c-tagging neural network variable. The effect of the detector design variation increases with increasing purity (harder c-tagging neural network cut). For the second set of points in the plot with purities about $18 \%$ and c-tagging efficiencies between $85 \%$ and $90 \%$, the variation of the signal efficiency and the number of We $\nu$ background events is given in the table after all selection cuts.

\begin{tabular}{l|c|c|c|c} 
Thickness & layers & signal efficiency (in \%) & $\mathrm{We} \nu / 210 \mathrm{k}$ & $\mathrm{We} \nu / 500 \mathrm{fb}^{-1}$ \\
\hline Single & $5(4)$ & $20.46(19.67)$ & $364(369)$ & $5322(5396)$ \\
Double & $5(4)$ & $20.32(19.52)$ & $366(385)$ & $5352(5630)$ \\
& & \pm 0.18 & \pm 19 & \pm 280
\end{tabular}

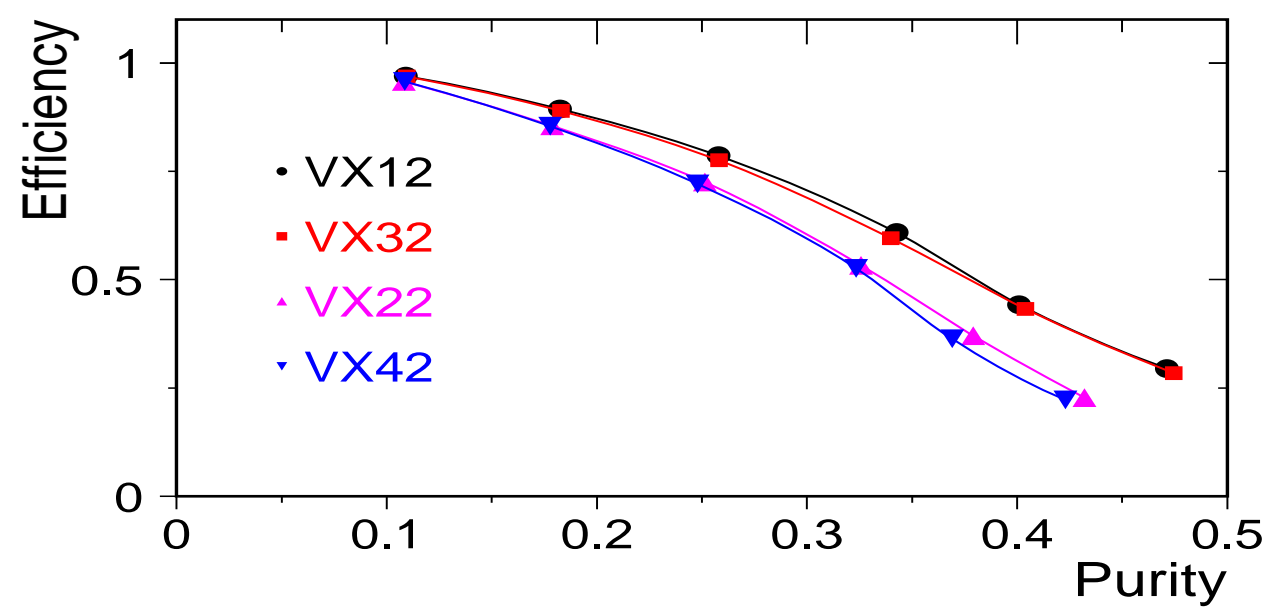

Figure 1. Left: scalar top c-tagging efficiency and purity with $\mathrm{We} \nu$ background for different detector designs. The $\mathrm{VX}_{12}$ curve is for a detector design with 5 layers (innermost at $15 \mathrm{~mm}$ ) and single density, curve $\mathrm{VX}_{22}$ is for a detector design with 4 layers (innermost at $26 \mathrm{~mm})$. Curves $\mathrm{VX}_{32}$ and $\mathrm{VX}_{42}$ are for double density $\left(0.128 \% X_{0}\right.$ radiation length per layer) with 4 and 5 layers, respectively. Right: expected signal efficiency and number of We $\nu$ background events for the four detector designs. The statistical uncertainties are also given.

\section{Results}

These results for small visible energy $(\Delta m=10 \mathrm{GeV})$ lead to the same observation as for large visible energy (SPS-5 scenario with $\Delta m=100.7 \mathrm{GeV}$ ). The radius of the innermost layer of the vertex detector has a large effect on the c-quark tagging performance. Curves $\mathrm{VX}_{12}$ and $\mathrm{VX}_{22}$ of Fig. 1 show the performance for radii 15 and $26 \mathrm{~mm}$, respectively. There is no significant effect on the c-quark tagging performance from doubling the material budget (e.g. curves $\mathrm{VX}_{12}$ and $\mathrm{VX}_{32}$ of Fig. 1). The increase of multiple scattering is not significant between single and double thickness $\left(0.128 \% X_{0}\right.$ radiation length per layer).

In order to quantitatively estimate the multiple scattering effect, the number of tracks per signal event and the visible energy have been determined. The minimum visible energy per 


\section{Linear Collider Workshop}

event is about $10 \mathrm{GeV}$ and the maximum number of tracks is about 20, therefore the minimum track energy is about $0.5 \mathrm{GeV}$. The analytical calculation of the multiple scattering angle is given by $\theta \approx 13.6 / P \cdot \sqrt{x / X_{0}}$, where the track momentum $P$ is given in MeV. The displacement at the interaction point is $d \approx R \theta$, where $R$ is the radius of the innermost layer of the vertex detector. For $P=500 \mathrm{MeV}, x / X_{0}=0.128 \%$ and $R=15 \mathrm{~mm}$, $d=15 \mu \mathrm{m}$. This small value compared to the flight distance of charm mesons explains the insignificant effect on the c-quark tagging from the multiple scattering increase by doubling the vertex detector layer thickness.

\section{Conclusions}

In conclusion, the studies with a small visible energy signal lead to the same results as in the previous study for large visible energy regarding the vertex detector design. A strong dependence on the detector performance on the radius of the innermost vertex detector layer has been observed, while doubling the material thickness has no significant effect on the c-quark tagging performance in scalar top studies at the ILC. The optimization of the radius of the innermost vertex detector layer will have to take into account the accelerator background which depends on the machine optics and the collimation system.

\section{Acknowledgements}

AS would like to thank the organizers of the workshop for making this presentation possible.

\section{References}

[1] M. Carena, A. Finch, A. Freitas, C. Milstène, H. Nowak and A. Sopczak, Phys. Rev. D72 (2005) 115008.

[2] C. Milsténe and A. Sopczak, Econf C0508141:ALCPG1431, 2005.

[3] A. Finch, H. Nowak and A. Sopczak, "CCD Vertex Detector Charm-Tagging Performance in Studies of Scalar Top Quark Decays", Proc. of the Int. Conference on Linear Colliders (LCWS 04), Paris, France, 19-24 April 2004.

[4] A. Sopczak, M. Carena, A. Finch, A. Freitas, C. Milsténe, H. Nowak, "Scalar Top Quark Studies with Various Visible Energies", presented at the 13th Int. Conference on Supersymmetry and Unification of Fundamental Interactions (SUSY'05), Durham, UK, July 18-23, 2005.

[5] T. Sjöstrand et al., Comput. Phys. Commun. 135, 238 (2001).

[6] A. Sopczak, in PHYSICS AT LEP2, "Event Generators for Discovery Physics", CERN Yellow Report CERN 96-01.

[7] A. Finch, H. Nowak and A. Sopczak, contributed paper EPS370, Int. Conference on HighEnergy Physics (HEP 2003), Aachen, Germany, 17-23 July 2003 [LC Note LC-PHSM-2003075].

[8] M. Pohl and H. J. Schreiber, hep-ex/0206009.

[9] M. Berggren, Simulation Grand Vitesse (SGV), http://berggren.home.cern.ch/berggren/sgv.html

[10] A. Freitas, D. J. Miller and P. M. Zerwas, Eur. Phys. J. C 21 (2001) 361; A. Freitas, A. von Manteuffel and P. M. Zerwas, Eur. Phys. J. C 34 (2004) 487.

[11] F. Yuasa et al., Prog. Theor. Phys. Suppl. 138, 18 (2000).

[12] E. Boos et al. [CompHEP Collaboration], Nucl. Instrum. Meth. A 534, 250 (2004).

[13] T. Kuhl, "Hadronic branching ratio of a SM-like Higgs boson at a future linear collider", Proc. of the Int. Conference on Linear Colliders (LCWS 04), Paris, France, 19-24 April 2004. 